Received: 26.09 .2018

Revised: 18.10 .2018

Accepted: 02.11.2018

DOI: $10.17804 / 2410-9908.2018 .6 .027-036$

\title{
STYDYING THE INFLUENCE OF THE NATURE OF THE FILLER ON THE MECHANICAL PROPERTIES OF THE DER-330 EPOXY RESIN
}

\author{
T. A. Brusentseva ${ }^{1, a^{*}}$, E. O. Smirnova ${ }^{2, \text { b) }}$, and I. A. Veretennikova ${ }^{2, c)}$ \\ ${ }^{I}$ S. A. Khristianovich Institute of Theoretical and Applied Mechanics, \\ Siberian Branch of the Russian Academy of Sciences, 4/1 Institutskaya St., Novosibirsk, 630090, Russian Federation \\ ${ }^{2}$ Institute of Engineering Science, Ural Branch of the Russian Academy of Sciences, \\ 34 Komsomolskaya St., Ekaterinburg, 620049, Russian Federation

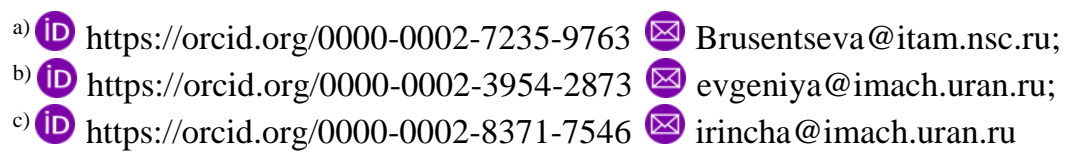 \\ *Corresponding author. E-mail: Brusentseva@itam.nsc.ru \\ Address for correspondence: ul. Institutskaya, 4/1, Novosibirsk, 630090, Russian Federation \\ Tel.: +7 (383) 3304275
}

The purpose of this paper is to investigate the effect of the nature of various fillers on the mechanical properties of the DER-330 epoxy resin. The A-380 and A-200 hydrophilic silica powders, alumina-based powders in the form of spherical particles and nanofibers, and aluminum nitride powder are used as fillers. The mass content of nanoadditives in the matrix ranges from 0 to $4 \%$. In the first series of experiments, the influence of the nature of the filler on the mechanical properties of the DER-330 epoxy resin under conditions of three-point bending is investigated. In the second part of the study, the influence of the nature of the filler on the hardness and the normal modulus of elasticity is investigated by the microindentation method. It has been experimentally established that the investigated solid nanopowders reinforce the epoxy resin provided that they are nanosized, effectively dispersed and uniformly distributed in the matrix. In addition, for each filler, there is an optimal content enabling the material to exhibits maximum strength characteristics.

Keywords: epoxy resin, nanofiller, modulus of elasticity, stress at failure, microindentation.

\section{Acknowledgment}

The work was performed within state assignment No. AAAA-A17-117030610134-9 (specimen preparation and experimenting under conditions of three-pint bending) and supported by RFBR grant No. 16-08-01154 A (indentation experiments).

\section{References}

1. Kickelbick G. Concepts for the incorporation of inorganic building blocks into organic polymers on a nanoscale. Progress in Polymer Science, 2003, vol. 28, iss. 1, pp. 83-114. DOI: 10.1016/S0079-6700(02)00019-9.

2. Rahman A., Ali I., Al Zahrani S.M., Eleithy R.H. A review of the applications of nanocarbon polymer composites. Nano, 2011, vol. 06, no. 03, pp 185-203. DOI: 10.1142/S179329201100255X.

3. Hashin Z. The elastic module of heterogeneous materials. J. Appl. Mech., 1962, vol. 29, pp. 143-150.

4. Ishai O., Cohen L.J. Strain-rate dependence of the elastic modulus of filled and porous epoxy composites. Int. J. Mech. Sci., 1967, vol. 9, iss. 9, pp. 605-608. DOI: 10.1016/0020-7403(67)90065-3. 
5. Paul B. Prediction of elastic constants of multiphase materials. Trans. AIME, 1960, vol. 218, pp. $36-41$.

6. Omrani A., Rostami A.A. Understanding the effect of nano-Al2O3 addition upon the properties of epoxy-based hybrid composites. Materials Science and Engineering A, 2009, vol. 517, iss. 1-2, pp. 185-190. DOI: 10.1016/j.msea.2009.03.076.

7. Chen C.H., Jian J.Y., Yen F.S. Preparation and characterization of epoxy/ $\gamma$-aluminum oxide nanocomposites. Composites Part A: Applied Science and Manufacturing, vol. 40, no. 4, pp. 463-468. DOI: 10.1016/j.compositesa.2009.01.010.

8. Al-Turaif Hamad A. Effect of nano $\mathrm{TiO}_{2}$ particle size on mechanical properties of cured epoxy resin. Progress in Organic Coatings, 2010, vol. 69, no. 3, pp. 241-246. DOI: 10.1016/j.porgcoat.2010.05.011.

9. Adachi T., Osaki M., Araki W., Kwon S-C. Fracture toughness of nano- and micro-spherical silica-particle-filled epoxy composites. Acta Materialia, 2008, vol. 56, no. 9, pp. 2101-2109. DOI: 10.1016/j.actamat.2008.01.002.

10. Li H., Zhang Z., Ma X., Hu M., Wang X., Fan P. Synthesis and characterization of epoxy resin modified with nano-SiO2 and $\gamma$-glycidoxypropyltrimethoxy silane. Surf. Coat. Tech., 2007, no. 201, pp. 5269 - 5272. DOI: 10.1016/j.surfcoat.2006.07.143.

11. Hsieh T.H., Kinloch A.J., Masania K., Sohn L.J., Taylor A.C. The toughness of epoxy polymers and fibre composites modified with rubber microparticles and silica nanoparticles. J. Mater. Sci., 2010, vol. 45, pp. 1193-1210. DOI: 10.1007/s10853-009-4064-9.

12. Oliver W.C., Pharr G.M. An improved technique for determining hard-ness and elastic modulus using load-displacement sensing indentation experiments. Mater. Res., 1992, vol. 7, no. 6, pp. 1564-1583. DOI: 10.1557/JMR.1992.1564.

13. Brusentseva T.A., Filippov A.A., Fomin V.M., Malykhin E.V., Vaganova T.A. Influence of the nanosized filler nature on the mechanical properties of epoxy-anhydride polymer composites. Nanotechnologies in Russia, 2014, vol. 9, iss. 11-12, pp 638-644. DOI: 10.1134/S1995078014060068.

14. Lipatov Yu.S. Fiziko-khimicheskie osnovy napolneniya polimerov [Physico-Chemical Bases of Polymer Filling]. Moscow, Khimiya Publ., 1991. (In Russian).

15. Suvorov A.L., Dul'tseva L.D., Ovchinnikova G.I., Khrustaleva E.A., Ostanina N.Yu., Abramova V.I. Preparation and Properties of Polymers Derived from Epoxy Resins and Oligochelatotitanophenylenesiloxanes. Russian Journal of Applied Chemistry, 2003, vol. 76, no. 11, pp. 1844-1849. DOI: 10.1023/B:RJAC.0000018697.76047.67.

16. Hoebbel D., Nacken M., Schidt H. On the influence of metal alkoxides on the epoxide ringopening and condensation reactions of 3-glycidoxypropyltrimethoxysilane. J. Sol-Gel Sci. Technol., 2001, vol. 21, no. 3, pp. 178-187. DOI: 10.1023/A:1011274301896. 
Подана в журнал: 26.09.2018

УДК 678.01

DOI: $10.17804 / 2410-9908.2018 .6 .027-036$

\title{
ИССЛЕДОВАНИЕ ВЛИЯНИЯ ПРИРОДЫ НАПОЛНИТЕЛЯ НА МЕХАНИЧЕСКИЕ ХАРАКТЕРИСТИКИ ЭПОКСИДНОЙ СМОЛЫ DER-330
}

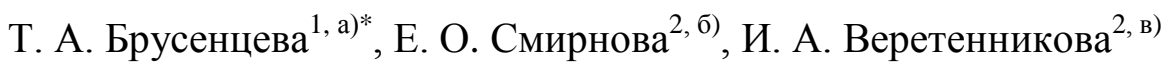 \\ ${ }^{1}$ Федеральное государственное бюджетное учреждение науки \\ Институт теоретической и прикладной механики им. С.А. Христиановича СО РАН, \\ 630090, ул. Институтская, 4/1, г. Новосибирск, Российская Федерация \\ ${ }^{2}$ Федеральное государственное бюджетное учреждение науки \\ Институт машиноведения Уральского отделения Российской академии наук, \\ 620049, ул. Комсомольская, 34, г. Екатеринбург, Российская Федераџия \\ a) (iD https://orcid.org/0000-0002-7235-9763 Brusentseva@itam.nsc.ru; \\ б) (iD https://orcid.org/0000-0002-3954-2873 evgeniya@imach.uran.ru; \\ в) iD https://orcid.org/0000-0002-8371-7546 irincha@imach.uran.ru \\ *Ответственный автор. Эл. почта: Brusentseva@itam.nsc.ru \\ Адрес для переписки: ул. Институтская, 4/1, 630090, г. Новосибирск, Российская Федерация \\ Тел.: +7 (383) 330-42-75
}

Исследовано влияние природы различных наполнителей на механические характеристики эпоксидной смолы DER-330. В качестве наполнителей использованы гидрофильные порошки оксида кремния A-380 и A-200, порошки на основе оксида алюминия в виде сферических частиц и нановолокон, порошок нитрида алюминия. Массовое содержание нанодобавок в матрице варьировалось от 0 до $4 \%$. В первой серии экспериментов исследовано влияние природы наполнителя на механические характеристики эпоксидной смолы DER-330 в режиме трехточечного изгиба. Во второй части работы методом микроиндентирования исследовано влияние природы наполнителя на твердость и нормальный модуль упругости. Экспериментально установлено, что исследуемые твердые нанопорошки усиливают эпоксидную смолу при условии, что они имеют наноразмеры, эффективно диспергированы и однородно распределены в матрице. Кроме того, для каждого наполнителя существует оптимальное содержание, при котором материал проявляет максимальные прочностные характеристики.

Ключевые слова: эпоксидная смола, нанонаполнитель, модуль упругости, напряжение при разрушении, микроиндентирование.

\section{1. Введение}

Изделия и конструкции из полимерных материалов широко используются в повседневной жизни и строительстве. Постоянное развитие технологий способствует расширению этого списка и возможностей применения данного материала. Перспективным направлением повышения эксплуатационных характеристик эпоксидных смол является использование добавок различной природы, поскольку они способствуют улучшению тепловых, механических, реологических, электрических и оптических свойств полимерных материалов [1, 2]. В частности, увеличение жесткости материала пропорционально количеству жестких частиц стеклянных шариков [3], в то время как добавление каучуковых частиц приводит к уменьшению жесткости[4], что подтверждается как в экспериментальных, так и в теоретических исследованиях [5-7]. Природа и размеры наполнителей также оказывают существенное влияние на характеристики полимерного материала. Например, использование 2 \% нанопорошка 
$\mathrm{Al}_{2} \mathrm{O}_{3}$ увеличивает модуль упругости эпоксидного композита на $23 \%$ [8, 9]. Авторы работы [10] изготовили образцы гетерогенного материала на основе эпоксидной смолы, армированного наноразмерными частицами $\mathrm{TiO}_{2}$, и получили, что максимум напряжения при растяжении достигается при 3 \% объемной концентрации наполнителя.

Появление углеродных нанотрубок (УНТ), фуллеренов, наноалмазов и активное изучение их свойств, привело к большому числу работ, посвященных изучению механических, электро- и теплопроводных свойств гетерогенных материалов с добавлением углеродных наночастиц. Это связано с тем, что физические свойства наноразмерных частиц существенно отличаются от свойств обычных материалов, поскольку большая часть атомов находится на поверхности частицы. Из результатов работ [11-13] следует, что концентрация диоксида кремния, когда достигается максимум механических характеристик эпоксидного композита, может различаться на порядки в зависимости от размера и формы частиц, присутствия связующего агента. Следовательно, при наличии нескольких варьируемых параметров наполнителя их влияние на механические характеристики гетерогенного материала носит комплексный, а не аддитивный характер. По этой причине, предсказание свойств различных по составу гетерогенных материалов на основе эпоксидной смолы является пока неразрешимой задачей и требует проведения комплексных экспериментальных исследований. Таким образом, цель работы - исследование влияния природы различных наполнителей на механические характеристики эпоксидной смолы DER-330. В качестве методов испытания выбраны трехточечный изгиб и индентирование.

\section{2. Материалы и оборудование}

В качестве полимерной матрицы для нанокомпозитного материала использовали эпоксидную смолу DER-330 и отвердитель изометилтетрагидрофталевого ангидрида (ИМТГФА), используемые в качестве эпоксидного связующего для создания перспективных гетерогенных материалов различного функционального назначения. Массовое содержание нанодобавок в матрице варьировалось от 0 до $4 \%$. В качестве наполнителей использовали нанодисперсные порошки:

1. А-380 (E1) - аэросил, гидрофильный порошок окиси кремния, $\mathrm{SiO}_{2}>99,8 \%$; $\mathrm{Al}_{2} \mathrm{O}_{3}<0,05 \% ; \mathrm{Fe}_{2} \mathrm{O}_{3}<0,003 \% ; \mathrm{TiO}_{2}<0,03 \% ; \mathrm{HCl}<0,025 \%$. Среднечисленный размер 7 нм, удельная площадь поверхности $380 \mathrm{~m}^{2} / \Gamma$.

2. A200 (E2) - аэросил, гидрофильный порошок окиси кремния, $\mathrm{SiO}_{2}>99,8$ \%; $\mathrm{Al}_{2} \mathrm{O}_{3}<0,05 \% ; \mathrm{Fe}_{2} \mathrm{O}_{3}<0,003 \% ; \mathrm{TiO}_{2}<0,03 \% ; \mathrm{HCl}<0,025 \%$. Среднечисленный размер 14 нм, удельная площадь поверхности $200 \mathrm{~m}^{2} / \Gamma$.

3. $\mathrm{Al}_{2} \mathrm{O}_{3}$ (E3) - окись алюминия, модифицированная винилтриметоксисиланом $\mathrm{Si}(\mathrm{OMe})_{3} \mathrm{C}_{2} \mathrm{H}_{3}$. Среднечисленный размер 65-67 нм, удельная площадь поверхности $22 \mathrm{~m}^{2} / \Gamma$.

4. $\mathrm{HBAl}_{2} \mathrm{O}_{3}$ (E4) - нановолокна окиси алюминия, модифицированные винилтриметоксисиланом $\mathrm{Si}(\mathrm{OMe})_{3} \mathrm{C}_{2} \mathrm{H}_{3}$. Удельная площадь поверхности $425 \mathrm{~m}^{2} / \Gamma$.

5. AlN (E5) - нитрид алюминия, AIN $82 \%$; Al(0) 3 \%. Среднечисленный размер 40-50 нм, удельная площадь поверхности $43 \mathrm{~m}^{2} / \Gamma$.

Прочностные характеристики исследованы в режиме трехточечного изгиба. Эксперименты проводили на машине для испытания материалов Zwick/Roell Z005 по стандарту испытаний $A S T M D$ 790. Для эксперимента изготавливали прямоугольные образцы $4 \times 10 \times 80$ мм³ $^{3}$. Нагружение (трехточечный изгиб с разгрузкой) задавали перемещением подвижной траверсы с постоянной скоростью 5 \%/мин, деформацию измеряли датчиком перемещения траверсы, приложенную силу - встроенным датчиком силы. Расстояние между опорами 65 мм. Эксперименты проводили до $8 \%$ деформации. Приведены усредненные данные испытаний не менее 5 образцов. Погрешность измерений модуля упругости составляет $\leq 3 \%$, предела прочности $-\leq 10 \%$. 
Эксперименты по инструментальному индентированию [14] проводили с помощью комплекса NanoTriboindentor TI 950. При индентировании использовали треугольный режим нагружения, при котором происходит линейный рост нагрузки и разгрузки с заданным постоянным временем. Индентирование проводили с использованием трехгранной пирамиды Берковича. Первичную обработку результатов испытаний осуществляли с помощью программного обеспечения прибора по ISO 14577-1:2002. В качестве характеризующих параметров были выбраны твердость $H$ и приведенный модуль упругости $E_{\mathrm{r}}$.

\section{3. Экспериментальные исследования на трехточечный изгиб}

Исследовано влияние природы наполнителя на механические характеристики эпоксидной смолы DER-330. В качестве наполнителей использованы гидрофильные порошки серии E1-E5. Массовое содержание нанодобавок в матрице варьировали от 0 мас. \% до 4 мас. \%. Исследованы зависимости механических свойств эпоксидных композитов в режиме трехточечного изгиба от концентрации и природы наполнителя. На рис. 1 и 2 представлены концентрационные зависимости модуля Юнга и напряжения разрушения гетерогенного материала с различными наполнителями в зависимости от концентрации. Величина модуля Юнга и напряжение при разрушении базового образца эпоксидной смолы DER-330 были получены ранее в работе [15] и составляют $E_{0}=3460 \mathrm{MПа} \mathrm{и} \sigma_{0}=155$ МПа соответственно. В работе рассматриваются отнесенные значения $E / E_{0}$ и $\sigma / \sigma_{0}$.

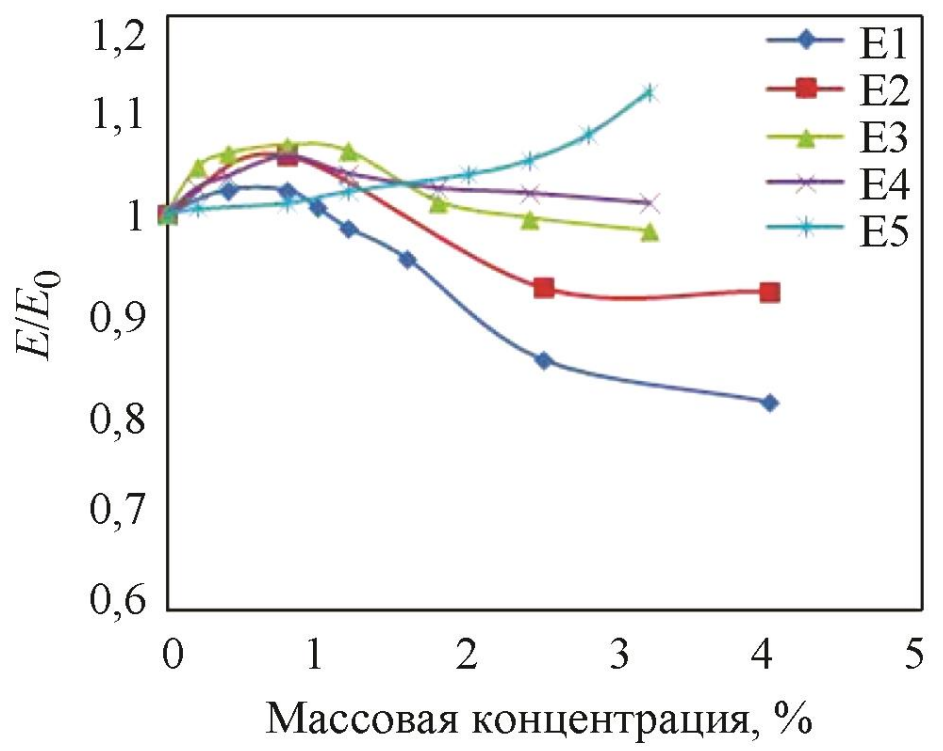

Рис. 1. Зависимость отнесенного модуля Юнга от массовой концентрации наполнителей

Качественный характер зависимости модуля Юнга от концентрации наполнителя для композитов E1-E4 подобен. До достижения концентрации 1,2 мас. \% модуль упругости для всех этих образцов растет, достигая максимума, и затем плавно снижается. Максимальное увеличение на $7 \%$ реализуется при использовании добавки $\mathrm{Al}_{2} \mathrm{O}_{3}$ (E3) в концентрации 0,8 мас. \%. Для образцов Е2 и Е4 модуль упругости при концентрации 1,2 мас. \% выше на $6 \%$, чем $E_{0}$ для чистой смолы соответственно. Дальнейшее увеличении концентрации вводимых добавок для образцов Е3 и Е4 приводит к тому, что при концентрации от 2,5 до 3,2 мас. \% значения $E$ находятся на уровне $E_{0}$. В свою очередь для образцов Е1 и Е2 при введении добавки больше 1,2 мас. \% наблюдается резкое падение значений модуля упругости на 20 и $8 \%$ соответственно. Таким образом, для каждого наполнителя существует оптимальное содержание, при котором материал проявляет максимальные прочностные характеристики. Внесение наполнителя выше оптимальной концентрации приводит к образованию агломера- 
тов, которые являются концентраторами напряжений, и тем самым способствуют разрушению композита при меньших нагрузках. Характер зависимости для композита с порошком нитрида алюминия (Е5) отличается от других: модуль упругости монотонно растет и достигает максимума при концентрации 3,2 мас. \% (увеличение на 12 \%).

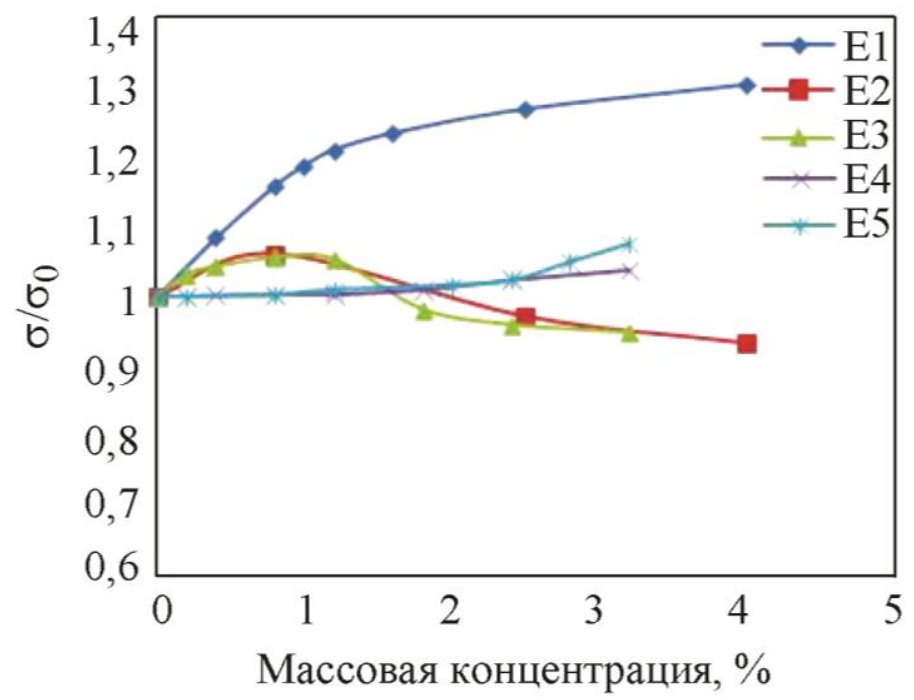

Рис. 2. Зависимость напряжения разрушения при трехточечном изгибе от массовой концентрации порошков

При добавке частиц А-380 сразу наблюдается наибольший рост показателя напряжения разрушения по сравнению с другими образцами. Так, добавление 4 мас. \% частиц приводит к увеличению напряжения при разрушении на 30 \%. При добавке частиц $\mathrm{HB} \mathrm{Al}_{2} \mathrm{O}_{3}$ и $\mathrm{AlN}$ рост напряжения при разрушении происходит только при концентрации больше 2,5 \%. Для образцов с добавками Е2 и Е3 зависимости $\sigma / \sigma_{0}$ от массовой концентрации количественно и качественно практически совпадают. В диапазоне от 0 до 1 мас. \% происходит рост напряжений разрушения, затем в диапазоне от 1 до 2 мас. \% плавно уменьшается до $\sigma_{0}$, затем при 2-4 мас. \% уменьшается на 8 \%. Таким образом, для всех добавок наблюдается неоднозначная связь между массовой концентрацией введенной добавки и полученными механическими характеристиками (модулем Юнга и напряжением при разрушении).

\section{4. Эксперименты по микроиндентированию и их результаты}

Для эксперимента по индентированию были выбраны следующие образцы: чистая смола DER-330, образцы с добавками A-380 (0,8 и 1,2 мас. \%), $\mathrm{Al}_{2} \mathrm{O}_{3}(0,4$ и 2,4 мас. \%) и AlN $(2,4$ мас. \%). Данные концентрации являются оптимальными и приводят к улучшению механических характеристик гетерогенного материала.

В первой серии экспериментов исследовали оценку степени равномерности распределения наполнителя и влияние продолжительности цикла нагружения на твердость и нормальный модуль упругости. Максимальная нагрузка при всех испытаниях составляла $0,5 \mathrm{H}$, время нагружения $\left(t_{\mathrm{H}}\right)-5 ; 10 ; 20 ; 30 ; 40 ; 50$ и 60 с (табл. 1$)$.

Для каждого времени нагружения было выполнено по 6 испытаний, результаты которых усреднялись. На основании проведенных тестов установлено, что разброс в значениях твердости материала для каждого образца остается в пределах $5 \%$, что свидетельствует о достаточно равномерном распределении частиц в полимерной матрице. На рис. 3 приведены усредненные значения твердости $H$ (рис. $3 a$ ) и нормального модуля упругости $E_{\mathrm{r}}$ (рис. $3 \sigma$ ), определяемого методом инструментального индентирования, в зависимости от времени

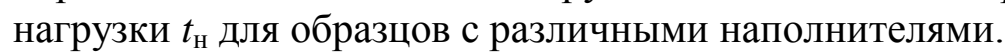


Таблица 1 - Результаты экспериментов по микроиндентированию

\begin{tabular}{|c|c|c|c|c|c|c|c|c|c|c|c|c|}
\hline & \multicolumn{2}{|c|}{$\begin{array}{c}\text { DER-330 } \\
0 \%\end{array}$} & \multicolumn{2}{c|}{$\begin{array}{c}\mathrm{A}-380 \\
0,8\end{array}$} & \multicolumn{2}{c|}{$\begin{array}{c}\mathrm{A}-380 \\
1,2 \%\end{array}$} & \multicolumn{2}{c|}{$\begin{array}{c}\mathrm{Al}_{2} \mathrm{O}_{3} \\
0,4\end{array}$} & \multicolumn{2}{c|}{$\begin{array}{c}\mathrm{Al}_{2} \mathrm{O}_{3} \\
2,4 \%\end{array}$} & \multicolumn{3}{|c|}{$\begin{array}{c}\mathrm{AlN} \\
2,4 \%\end{array}$} \\
\hline$t_{\mathrm{H}}$ & $\begin{array}{c}E_{\mathrm{r}} \\
(\mathrm{GPa})\end{array}$ & $\begin{array}{c}H \\
(\mathrm{GP})\end{array}$ & $\begin{array}{c}E_{\mathrm{r}} \\
(\mathrm{GPa})\end{array}$ & $\begin{array}{c}H \\
(\mathrm{GP})\end{array}$ & $\begin{array}{c}E_{\mathrm{r}} \\
(\mathrm{GPa})\end{array}$ & $\begin{array}{c}H \\
(\mathrm{GPa})\end{array}$ & $\begin{array}{c}E_{\mathrm{r}} \\
(\mathrm{GPa})\end{array}$ & $\begin{array}{c}H \\
(\mathrm{GP})\end{array}$ & $\begin{array}{c}E_{\mathrm{r}} \\
(\mathrm{GPa})\end{array}$ & $\begin{array}{c}H \\
(\mathrm{GP})\end{array}$ & $\begin{array}{c}E_{\mathrm{r}} \\
(\mathrm{GPa})\end{array}$ & $\begin{array}{c}H \\
(\mathrm{GP})\end{array}$ \\
\hline 5 & 5,35 & 0,43 & 5,69 & 0,45 & 5,65 & 0,45 & 5,53 & 0,47 & 5,29 & 0,44 & 5,67 & 0,44 \\
\hline 10 & 5,27 & 0,41 & 5,49 & 0,43 & 5,59 & 0,43 & 5,45 & 0,45 & 5,12 & 0,42 & 5,60 & 0,42 \\
\hline 20 & 5,22 & 0,39 & 5,44 & 0,42 & 5,54 & 0,41 & 5,40 & 0,43 & 5,07 & 0,40 & 5,55 & 0,40 \\
\hline 30 & 5,20 & 0,38 & 5,41 & 0,42 & 5,53 & 0,40 & 5,37 & 0,42 & 5,04 & 0,40 & 5,54 & 0,39 \\
\hline 40 & 5,13 & 0,37 & 5,42 & 0,40 & 5,54 & 0,40 & 5,41 & 0,42 & 5,03 & 0,39 & 5,54 & 0,39 \\
\hline 60 & 5,16 & 0,37 & 5,47 & 0,40 & 5,43 & 0,40 & 5,40 & 0,40 & 5,01 & 0,38 & 5,52 & 0,38 \\
\hline
\end{tabular}

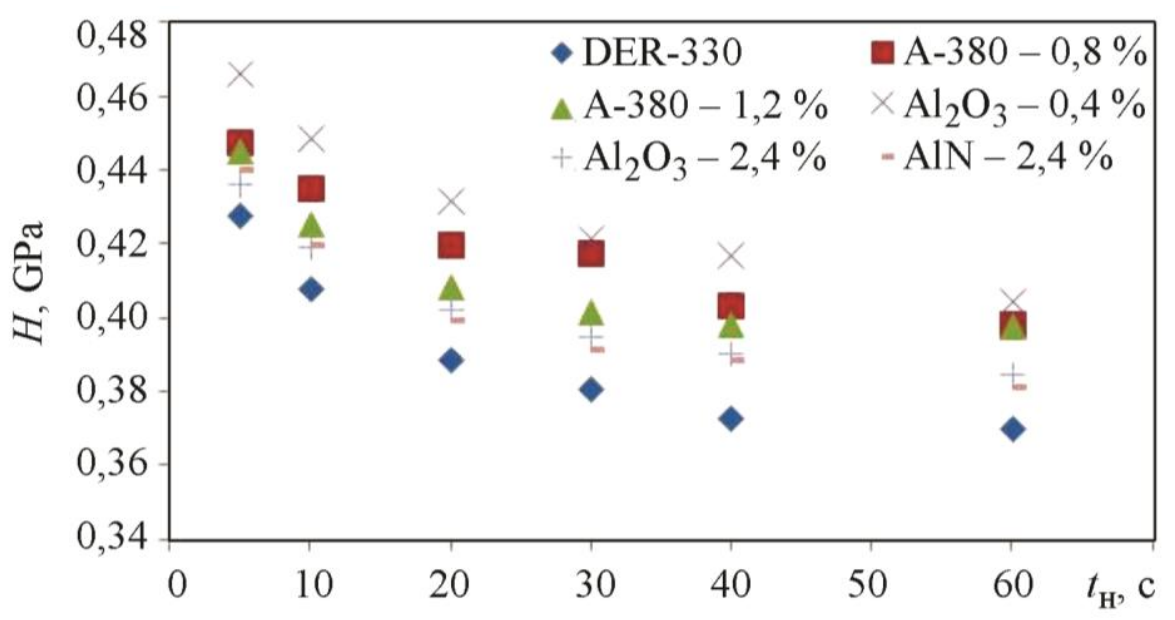

$a$

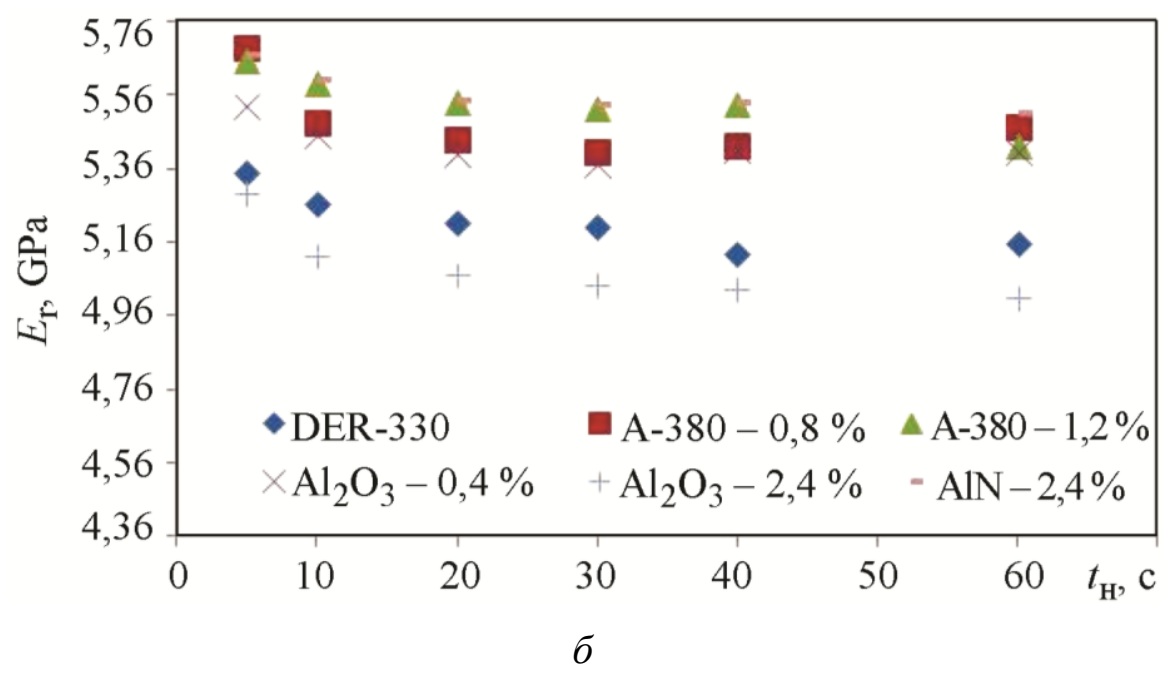

Рис. 3. Влияние времени нагружения на твердость (a) и приведенный модуль (б) для образцов

На рис. 3 а сохраняется общий вид зависимости твердости от времени нагружения в независимости от введения добавки. В диапазоне $t_{\mathrm{H}}$ от 1 до 40 с все образцы проявляют вязкие свойства, выражающиеся в уменьшении твердости при уменьшении скорости приложе- 
ния нагрузки. Для эпоксидной смолы DER-330 значение твердости снижается на 14 \%, для образцов, наполненных $\mathrm{AlN}$ - на $13 \%$, для $\mathrm{SiO}_{2}, \mathrm{Al}_{3} \mathrm{O}_{2}-$ на 12 \%. Дальнейшее увеличение времени $t_{\mathrm{H}}$ оказывает незначительное влияние на величину твердость для всех образцов.

Добавки оказывают влияние на получаемые значения твердости (табл. 2). Данные приведены для $t_{\mathrm{H}}=40 \mathrm{c}$, считается что при этом времени нагружения получаемые значения стабилизируются и дальнейшее увеличение времени нагружения не оказывает значительного влияния на твердость. Твердость нанонаполненного композита во всех случаях выше, чем у чистой эпоксидной смолы. Большее значение твердости у образцов эпоксидной смолы с нанопорошком $\mathrm{Al}_{2} \mathrm{O}_{3}$ с содержанием $0,4 \%-0,42$ ГПа. В свою очередь при увеличении содержания данного порошка до 2,4 \% получаемые значения твердости оказываются ниже 0,39 ГПа. Для эпоксидной смолы с нанопорошком диоксида кремния в концентрациях 0,8 \% и $1,2 \%$ значения твердости оказываются на одном и том же уровне 0,4 ГПа. Введение в эпоксидную смолу нановолокон AIN при концентрации 2,4 \% приводит к незначительному увеличению твердости - до 0,39 ГПа.

Максимум значений приведенного модуля упругости достигается при добавлении в матрицу наполнителя AlN и A-380, модуль увеличивается на 7,5 \%.

Таблица 2 - Значения твердости и приведенного модуля упругости

\begin{tabular}{|l|c|c|c|c|c|c|}
\hline & DER-330 & $\mathrm{A}-380$ & $\mathrm{~A}-380$ & $\mathrm{Al}_{2} \mathrm{O}_{3}$ & $\mathrm{Al}_{2} \mathrm{O}_{3}$ & $\mathrm{AlN}$ \\
\hline Массовая концентрация, \% & 0 & 0,8 & 1,2 & 0,4 & 2,4 & 2,4 \\
\hline$H(\mathrm{GPa})$ & 0,37 & 0,40 & 0,40 & 0,42 & 0,39 & 0,39 \\
\hline$E_{\mathrm{r}}(\mathrm{GPa})$ & 5,13 & 5,42 & 5,54 & 5,41 & 5,03 & 5,54 \\
\hline
\end{tabular}

На модуль упругости, полученный при индентировании, оказывает существенное влияние размер частиц: чем меньше размеры, тем модуль упругости выше. Кроме того, наблюдаемые явления зависимости твердости и приведенного модуля упругости от состава вводимого модификатора свидетельствуют о том, что оксидный модификатор в эпоксидной смоле проявляется не только как индифферентный наполнитель, изменяющий прочностные свойства материала в соответствии с классической теорией адсорбции полимеров [14], но и в некоторых случаях как реактивный компонент, формирующий определенный набор межмолекулярных взаимодействий, в том числе ковалентное взаимодействие $[15,16]$.

Таким образом, используемые наполнители различаются химическими свойствами, при этом они имеют общее - наномасштабный размер частиц. Экспериментально установлено, что исследуемые твердые нанопорошки усиливают эпоксидную смолу при условии, что они имеют наноразмеры и они эффективно диспергированы и однородно распределены в матрице.

\section{5. Заключение}

Проведены исследования зависимости механических характеристик эпоксидной смолы DER-330 от природы и концентрации наполнителя. Установлено, что введение наночастиц оксида кремния в количестве 1,2 \% от массы связующего приводит к увеличению напряжения при разрушении на $30 \%$. Введение 3,2 мас. \% нитрида алюминия приводит к росту модуля упругости на $12 \%$ при увеличении напряжения при разрушении на $7 \%$. Разброс в значениях твердости материала для каждого образца в пределах $5 \%$, что свидетельствует о достаточно равномерном распределении частиц в полимерной матрице. Показано, что в диапазоне времени нагружения от 1 до 40 с все образцы проявляют вязкие свойства, выражающиеся в уменьшении твердости при уменьшении скорости приложения нагрузки. Приведенный модуль упругости увеличивается на 7,5 \% при введении в эпоксидную смолу наполнителей AIN и A-380. Экспериментально установлено, что при оптимальной концентрации 1,2 \% твердые нанопорошки оксида кремния, нитрида алюминия 
и оксида алюминия усиливают эпоксидную смолу при условии, что они имеют наноразмеры и равномерно распределены в матрице.

\section{Благодарность}

Работа выполнена в части изготовления образиов и проведения экспериментов в режиме трехточечного изгиба в рамках государственного задания № AAAA-A17117030610134-9, в части проведения экспериментов по индентированию - при поддержке гранта РФФИ № 16-08-01154 A.

\section{Литература}

1. Kickelbick G. Concepts for the incorporation of inorganic building blocks into organic polymers on a nanoscale // Progress in Polymer Science. - 2003. - Vol. 28, iss. 1. - P. 83-114. DOI: 10.1016/S0079-6700(02)00019-9.

2. A review of the applications of nanocarbon polymer composites / A. Rahman, I. Ali, S. M. Al Zahrani, R. H. Eleithy // Nano. - 2011. - Vol. 06, no. 03. - P. 185-203. DOI: $10.1142 / \mathrm{S} 179329201100255 \mathrm{X}$.

3. Hashin Z. The elastic module of heterogeneous materials // J. Appl. Mech. - 1962. Vol. 29. - P. 143-150.

4. Ishai O., Cohen L. J. Strain-rate dependence of the elastic modulus of filled and porous epoxy composites // Int. J. Mech. Sci. - 1967. - Vol. 9, iss. 9. - P. 605-608. - DOI: 10.1016/00207403(67)90065-3.

5. Paul B. Prediction of elastic constants of multiphase materials // Trans. AIME. - 1960. Vol. 218. - P. 36-41.

6. Omrani A., Rostami A. A. Understanding the effect of nano-A12O3 addition upon the properties of epoxy-based hybrid composites // Materials Science and Engineering A. - 2009. Vol. 517. - P. 185-190. - DOI: 10.1016/j.msea.2009.03.076.

7. Chen C. H., Jian J. Y., Yen F. S. Preparation and characterization of epoxy/ $\gamma$-aluminum oxide nanocomposites // Composites Part A: Applied Science and Manufacturing. - 2009. - Vol. 40, no. 4. - P. 463-468. DOI: 10.1016/j.compositesa.2009.01.010.

8. Al-Turaif Hamad A. Effect of nano TiO2 particle size on mechanical properties of cured epoxy resin // Progress in Organic Coatings. - 2010. - Vol. 69, no. 3. - P. 241-246. DOI: $10.1016 /$ j.porgcoat.2010.05.011.

9. Fracture toughness of nano- and micro-spherical silica-particle-filled epoxy composites / T. Adachi, M. Osaki, W. Araki, S-C. Kwon // Acta Materialia. - 2008. - Vol. 56, no. 9. P. 2101-2109. - DOI: 10.1016/j.actamat.2008.01.002.

10. Synthesis and characterization of epoxy resin modified with nano-SiO2 and $\gamma$-glycidoxypropyltrimethoxy silane / H. Li, Z. Zhang, X. Ma, M. Hu, X. Wang, P. Fan // Surf. Coat. Tech. - 2007. - No. 201. - P. 5269-5272. - DOI: 10.1016/j.surfcoat.2006.07.143.

11. The toughness of epoxy polymers and fibre composites modified with rubber microparticles and silica nanoparticles / T. H. Hsieh, A. J. Kinloch, K. Masania, L. J. Sohn, A. C. Taylor // J. Mater. Sci. - 2010. - Vol. 45. - P. 1193-1210. - DOI: 10.1007/s10853-009-4064-9.

12. Oliver W. C., Pharr G. M. An improved technique for determining hard-ness and elastic modulus using load-displacement sensing indentation experiments // Mater.Res. - 1992. - Vol. 7, no. 6. - P. 1564-1583. - DOI: 10.1557/JMR.1992.1564.

13. Influence of the nanosized filler nature on the mechanical properties of epoxy-anhydride polymer composites / T. A. Brusentseva, A. A. Filippov, V. M. Fomin, E. V. Malykhin, T. A. Vaganova // Nanotechnologies in Russia. - 2014. - Vol. 9, iss. 11-12. - P. 638-644. DOI: $10.1134 / \mathrm{S} 1995078014060068$.

14. Липатов Ю. С. Физико-химические основы наполнения полимеров. - М. : Химия, 1991. - 257 c. 
15. Preparation and Properties of Polymers Derived from Epoxy Resins and Oligochelatotitanophenylenesiloxanes / A. L. Suvorov, L. D. Dul'tseva, G. I. Ovchinnikova, E. A. Khrustaleva, N. Yu. Ostanina, V. I. Abramova // Russian Journal of Applied Chemistry. - 2003. - Vol. 76, no. 11. - P. 1844-1849. -DOI: 10.1023/B:RJAC.0000018697.76047.67.

16. Hoebbel D., Nacken M., Schidt H. On the influence of metal alkoxides on the epoxide ringopening and condensation reactions of 3-glycidoxypropyltrimethoxysilane // J. Sol-Gel Sci. Technol. - 2001. - Vol. 21, no. 3. - P. 178-187. - DOI: 10.1023/A:1011274301896. 